\title{
Probing localization and quantum geometry by spectroscopy
}

\author{
Tomoki Ozawa $\odot^{1}$ and Nathan Goldman ${ }^{2}$ \\ ${ }^{1}$ Interdisciplinary Theoretical and Mathematical Sciences Program, RIKEN, Wako, Saitama 351-0198, Japan \\ ${ }^{2}$ Center for Nonlinear Phenomena and Complex Systems, Université Libre de Bruxelles, Campus Plaine, CP 231, 1050 Brussels, Belgium
}

(Received 11 May 2019; revised manuscript received 11 June 2019; published 15 November 2019)

\begin{abstract}
The spatial localization of quantum states plays a central role in condensed-matter phenomena, ranging from many-body localization to topological matter. Building on the dissipation-fluctuation theorem, we propose that the localization properties of a quantum-engineered system can be probed by spectroscopy, namely, by measuring its excitation rate upon a periodic drive. We apply this method to various examples that are of direct experimental relevance in ultracold atomic gases, including Anderson localization, topological edge modes, and interacting particles in a harmonic trap. Moreover, inspired by a relation between quantum fluctuations and the quantum metric, we describe how our scheme can be generalized in view of extracting the full quantum-geometric tensor of many-body systems. Our approach opens an avenue for probing localization, as well as quantum fluctuations, geometry and entanglement, in synthetic quantum matter.
\end{abstract}

DOI: 10.1103/PhysRevResearch.1.032019

Introduction. Localization plays a central role in various branches of quantum physics. In the context of the solid state, the localization properties of electronic wave functions reflect the conductivity of materials and signal the existence of insulating regimes [1-6]. This important relation between transport and the spatial localization of quantum states was already emphasized in the seminal work by Anderson on disordered lattice systems [1]. More recently, the discovery of topological states of matter revealed an interesting interplay between topology and localization: The bulk-boundary correspondence guarantees the existence of robust boundary modes, which are localized at the edge of the material $[7,8]$. Another important development concerns the phenomenon of many-body localization (MBL), which is characterized by the absence of thermalization in many-body systems featuring disorder and interparticle interactions [9-11].

Quantum engineered systems, such as ultracold atomic gases or trapped ions, have recently emerged as novel platforms by which localization can be finely studied in a highly controlled environment. Anderson localization was observed in Bose-Einstein condensates, in various spatial dimensions, through the design of disordered potentials for neutral atoms [12-14]. Besides the control over the disorder strength, these quantum-engineered systems also allow for the possibility of tuning the interparticle interactions. Combining these two appealing features led to the first experimental observations of MBL in ultracold atomic gases $[15,16]$, which were soon followed by realizations in trapped ions [17] and in photonics [18]. While the localization length was directly measured in the context of Anderson localization [12,14], by imaging

Published by the American Physical Society under the terms of the Creative Commons Attribution 4.0 International license. Further distribution of this work must maintain attribution to the author $(s)$ and the published article's title, journal citation, and DOI. the spatial profile of the atomic cloud in situ, such direct signatures of localization remain challenging in the MBL regime (see Ref. [19] for correlation-length measurements in the MBL phase based on single-site resolution imaging).

In this Rapid Communication we introduce a method by which localization can be quantitatively studied in quantumengineered systems, without relying on any in situ imaging. Our approach builds on a universal relation between the localization of a quantum state and its spectroscopic response to an external periodic drive and therefore can be applied to many-body (interacting) systems. In fact, this relation between localization properties and dissipative responses can be traced back to the fluctuation-dissipation theorem [20-22], as was previously noticed in the solid state [5,23]. In this Rapid Communication we propose that measuring the excitation rate of a quantum-engineered system upon a periodic drive offers a practical scheme by which its localization properties can be precisely evaluated. In particular, this method can be readily applied to general many-body systems, in the presence of interactions and/or disorder, as we illustrate below through relevant examples. Our proposal builds on the general observation that excitation-rate measurements can be used to extract useful information on the underlying quantum states, as was recently illustrated in the context of topological Bloch bands [24-27].

Beyond the localization of particles in extended lattice geometries, our method can also be applied to study localization in confined systems. This asset is relevant to ultracold atomic gases in optical lattices, where the spread of the twobody wave function within each lattice site affects the effective interaction energy [28-31]. While detecting localization within a single site of an optical lattice requires sophisticated nanoscale microscopy [32,33], this property could be equally studied using the more practical spectroscopic probe introduced in this work.

Dissipation and fluctuations are united through the imaginary part of the generalized susceptibility [20-22]. 
Interestingly, it was noted that this response function is also deeply related to the geometry of quantum states, through the notion of the quantum (Fubini-Study) metric [23,34,35] (see Ref. [36] for a similar observation involving the quantum Fisher information, which captures the entanglement properties of many-body quantum states). In many-body systems, the quantum metric is defined over the parameter space spanned by twist angles associated with boundary conditions [23]. As a by-product of our proposal, we establish a protocol by which the full quantum geometry of many-body quantum systems (including the quantum metric and the many-body Berry curvature [37-39]) can be extracted from spectroscopic responses.

Excitation rates and localization. We first discuss how the excitation rate of a quantum system upon a periodic drive relates to the variance of the position operator. Let us assume that the system is initially in an eigenstate $|\alpha\rangle$ of a many-body Hamiltonian $\hat{H}$, which we consider to be nondegenerate and well separated from all other states in energy. ${ }^{1}$ We then act on the system with a time-periodic perturbation aligned along the $x$ direction so that the total Hamiltonian reads

$$
\hat{H}(t)=\hat{H}+2 E \hat{x} \cos (\omega t),
$$

where $\hat{x} \equiv \sum_{a} \hat{x}_{a}$ is the many-body position operator along the $x$ direction and $\hat{x}_{a}$ is the position operator for the $a$ th particle. At the lowest order in time-dependent perturbation theory, the excitation fraction is given by Fermi's golden rule [40]

$$
n_{\hat{x}}^{\mathrm{ex}}(\omega, t)=\frac{2 \pi E^{2}}{\hbar} t \sum_{\beta \neq \alpha}|\langle\beta|\hat{x}| \alpha\rangle|^{2} \delta^{(t)}\left(\epsilon_{\beta}-\epsilon_{\alpha}-\hbar \omega\right),
$$

where $\delta^{(t)}(\epsilon) \equiv(2 \hbar / \pi t) \sin ^{2}(\epsilon t / 2 \hbar) / \epsilon^{2}$ can be approximated by a Dirac distribution for sufficiently large $t$. It is convenient to introduce the excitation rate $\Gamma_{\hat{x}}(\omega) \equiv n_{\hat{x}}^{\mathrm{ex}}(\omega, t) / t$, which is the rate at which the system excites to other states $\beta \neq \alpha$. Inspired by Refs. [25-27], we consider performing a set of experiments for various values of the shaking frequency $\omega$, and integrating the resulting rates over $\omega$,

$$
\Gamma_{\hat{x}}^{\mathrm{int}} \equiv \int_{0}^{\infty} \Gamma_{\hat{x}}(\omega) d \omega=\frac{2 \pi E^{2}}{\hbar^{2}} \sum_{\beta \neq \alpha}|\langle\beta|\hat{x}| \alpha\rangle|^{2} .
$$

Using the completeness relation $\sum_{\beta}|\beta\rangle\langle\beta|=1$, we rewrite the sum above as

$$
\begin{aligned}
\sum_{\beta \neq \alpha}|\langle\beta|\hat{x}| \alpha\rangle|^{2} & =\sum_{\beta \neq \alpha}\langle\alpha|\hat{x}| \beta\rangle\langle\beta|\hat{x}| \alpha\rangle \\
& =\left(\left\langle\alpha\left|\hat{x}^{2}\right| \alpha\right\rangle-\langle\alpha|\hat{x}| \alpha\rangle^{2}\right) \equiv \operatorname{Var}(\hat{x}),
\end{aligned}
$$

which is nothing but the variance $\operatorname{Var}(\hat{x})$ of the operator $\hat{x}$. When combined with Eq. (2), this yields a relation between the integrated rate and the spatial variance

$$
\Gamma_{\hat{x}}^{\mathrm{int}}=\frac{2 \pi E^{2}}{\hbar^{2}} \operatorname{Var}(\hat{x}) \text {. }
$$

\footnotetext{
${ }^{1}$ The results presented in this work are still valid for degenerate states, as long as the position operator does not couple different states within the degenerate manifold.
}

This relation establishes a protocol by which the variance of the position operator can be measured in experiments, without detecting the position of the particles.

In fact, the relation between the integrated rate and the variance of an operator can be made more general. For any operator $\hat{A}$, the excitation rate upon the drive $\hat{V}(t)=$ $2 E \hat{A} \cos (\omega t)$ is related to the variance of this operator through $\Gamma_{\hat{A}}^{\text {int }}=\frac{2 \pi E^{2}}{\hbar^{2}} \operatorname{Var}(\hat{A})$. Hence, time modulation can be used as a universal probe for the variance of any operator. Moreover, noting that the variance of an operator is directly related to a quantum Fisher information [36], we point out that this probe also captures the multipartite entanglement properties of many-body quantum states.

Excitation rates can be measured in various ways in quantum-engineered systems (see Refs. [24,41-44] for distinct experimental methods applied to periodically driven atomic gases). In optical lattices, heating rates can be finely measured by monitoring the dynamical repopulation of Bloch bands through band mapping [24,41]. In cases where intraband transitions are relevant and difficult to resolve [45], one can optimize such measurements by applying a drive that simultaneously changes the internal state of the atoms [46]; the excited fraction can then be determined by counting the number of atoms in different internal states.

We point out that the result in Eq. (4) derives from the fluctuation-dissipation theorem. To see this, recall that the fluctuation associated with an operator $\hat{A}$, in a given state $|\alpha\rangle$, is related to the generalized susceptibility $\chi_{\hat{A} \hat{A}}(\omega)$ via the fluctuation-dissipation theorem [22]

$$
\left\langle\alpha\left|\hat{A}^{2}\right| \alpha\right\rangle=\frac{\hbar}{\pi} \int_{0}^{\infty} d \omega \operatorname{Im}\left[\chi_{\hat{A} \hat{A}}(\omega)\right],
$$

where we assumed $\langle\alpha|\hat{A}| \alpha\rangle=0$. In addition, the power absorbed $\mathcal{P}(\omega)$ upon a periodic drive $2 E \hat{A} \cos (\omega t)$ is related to the imaginary part of the generalized susceptibility [22]: $\mathcal{P}(\omega)=2 \omega E^{2} \operatorname{Im}\left[\chi_{\hat{A} \hat{A}}(\omega)\right]$. Noting that the excitation rate is defined as $\Gamma(\omega)=\mathcal{P}(\omega) / \hbar \omega$, we recover the relation $\Gamma_{\hat{A}}^{\text {int }}=$ $\frac{2 \pi E^{2}}{\hbar^{2}} \operatorname{Var}(\hat{A})$.

Anderson model. We first apply our method to the Anderson model [2,3], which describes a quantum particle moving in a one-dimensional disordered lattice; the hopping matrix element is denoted $J$ and the random (disordered) potential has values uniformly distributed within the interval $[-W / 2, W / 2] J$. The presence of disorder generates localized eigenstates: The envelope of the wave functions takes the form $\sim e^{-|x| / \xi}$ around their average position, where $\xi$ is the localization length. The spatial variance calculated from this naive exponentially decaying wave function $\operatorname{reads} \operatorname{Var}(\hat{x})=$ $\xi^{2} / 2$, which indeed provides a qualitatively accurate estimate (deviations due to finer structures in the wave functions are illustrated below). According to the scaling theory of the Anderson model [3], the localization length at zero energy scales as $\xi \approx 96 J^{2} / W^{2}$, in units of the lattice spacing; the corresponding variance $\operatorname{reads} \operatorname{Var}(\hat{x}) \approx 4608 J^{4} / W^{4}$ (see Fig. 1).

We now show that these localization properties can be detected through excitation-rate measurements [Eq. (4)]. For a given disorder realization, we start from an eigenstate of the model whose energy is close to zero and then numerically 


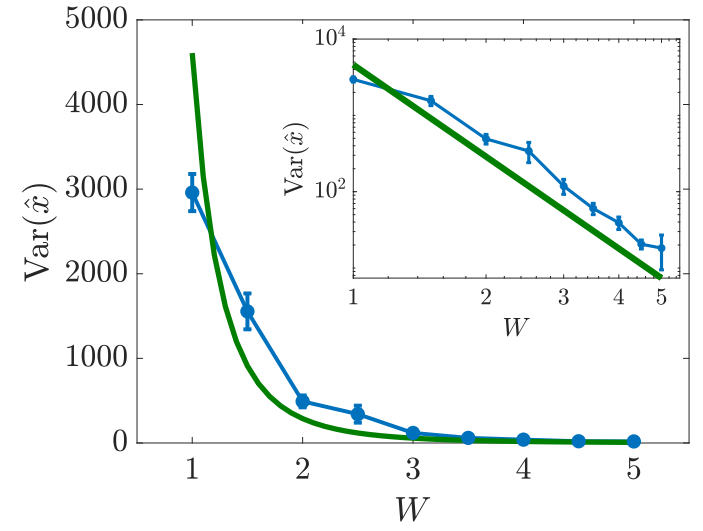

FIG. 1. Spatial variance $\operatorname{Var}(\hat{x})$ of zero-energy eigenstates as a function of the disorder strength $W$, as extracted from excitation-rate measurements [Eq. (4)]. Blue dots are numerical results obtained from 300 sites and an averaging over 20 disorder realizations (for each $W$ ). We used a modulation strength $E=0.001 \mathrm{~J}$ and an observation time of $t=5 \hbar / J$; the excitation rates were integrated over $\omega$ up to the value $5 J / \hbar$, using discrete steps of $0.01 J / \hbar$. The scaling-theory prediction $\operatorname{Var}(\hat{x}) \approx 4608 J^{4} / W^{4}$ is also displayed (green curve).

calculate the integrated excitation rate upon applying a periodic modulation [Eq. (1)]. The results are shown in Fig. 1, for various values of the disorder strength $W$, together with the scaling-theory prediction. Fitting the estimated variance with a power law, we obtain $\operatorname{Var}(\hat{x}) \approx 4700 / W^{3.4}$, which is indeed very close to the prediction $\sim 1 / W^{4}$; the main discrepancy is attributed to the finer structure of the wave function inside the envelop function $e^{-|x| / \xi}$. We note that the eigenstate considered in these calculations is not isolated in energy (there is no spectral gap in the thermodynamic limit); however, states with similar energies are well separated spatially, and hence they do not contribute to the excitation rate (see footnote 1).

Topological edges modes. As a second example, we consider the celebrated Su-Schrieffer-Heeger (SSH) model, a model exhibiting symmetry-protected topological edge modes $[47,48]$. This is a one-dimensional tight-binding model with alternating hopping strengths $t_{\text {weak }}$ and $t_{\text {strong }}$; here we assume

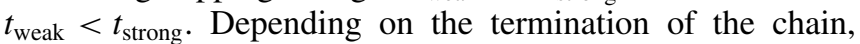
the boundaries can host localized (zero-energy) edge modes; these edge states are protected by topology, due to the chiral symmetry of the model [49]. Importantly, topological modes can also appear in the bulk of the chain [50] whenever the latter presents a defect (e.g., if the strengths of the hopping amplitudes $t_{\text {weak }}$ and $t_{\text {strong }}$ are locally interchanged); such a defect constitutes an interface between two regions associated with different topological invariants (winding numbers), which explains the presence of a zero-energy mode that is exactly localized at the interface [see a sketch of the setting and the corresponding localized wave function depicted in Figs. 2(a) and 2(b)]. We note that this wave function only takes nonzero values on every other site. Analytically, the overall decay of the wave function obeys $|\psi(x)|=\left(t_{\text {weak }} / t_{\text {strong }}\right)^{x / 2 a}$, where $x$ is the distance from the interface and $a$ is the lattice spacing. The spatial variance calculated from this analytical (a)
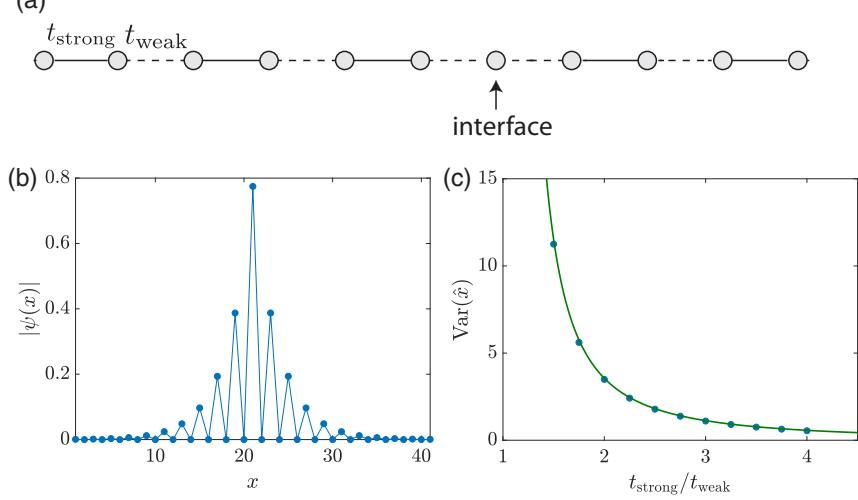

FIG. 2. (a) Schematics of the Su-Schrieffer-Heeger model, a onedimensional chain with alternating hopping strengths. We consider a situation where a topological interface is created in the middle of the chain, by introducing a defect (where hoppings are weak $t_{\text {weak }}$ on either side of a site). In this way, we obtain a zero-energy state that is localized at the interface. (b) Typical zero-energy mode localized at the interface. We used $t_{\text {strong }} / t_{\text {weak }}=2$. The interface is located at site 21 , where the wave function is maximal. (c) Variance $\operatorname{Var}(\hat{x})$ estimated from excitation-rate measurements upon linear driving, and the analytical value (6) as a function of $t_{\text {strong }} / t_{\text {weak }}$. We used $E=0.01 t_{\text {weak }} / a$ and an observation time $t=5 \hbar / t_{\text {weak }}$; the excitation rates were integrated over $\omega$ up to the value $5 t_{\text {weak }} / \hbar$, using discrete steps of $0.05 t_{\text {weak }} / \hbar$.

wave function reads

$$
\operatorname{Var}(\hat{x})=8\left(t_{\text {weak }} / t_{\text {strong }}\right)^{2} /\left[1-\left(t_{\text {weak }} / t_{\text {strong }}\right)^{2}\right]^{2} .
$$

We also assume that both ends of the chain are terminated with a strong link $\left(t_{\text {strong }}\right)$, so there is no additional edge state at the boundaries of the chain; in this setting, we have a single zero-energy mode, which is localized at the interface. We performed simulations of the driven SSH model, in view of numerically estimating $\operatorname{Var}(\hat{x})$ from the integrated excitation rates. The results are plotted in Fig. 2(c), where excellent agreement is shown between the excitation-rate measurement (dots) and the analytical result (solid line).

Interacting particles in a harmonic trap. We now consider a system of two particles of mass $m$, moving in a onedimensional harmonic trap of frequency $\Omega$ (see Ref. [51] for the single-particle case). We assume that the two particles are distinguishable ${ }^{2}$ and that they interact via a repulsive contact interaction $U \delta\left(\hat{x}_{1}-\hat{x}_{2}\right)$, with $U>0$. The interaction spreads out the ground-state wave function, as can be seen in the density distributions $n(x)$ depicted in Fig. 3(a) (see Ref. [52] for exact solutions). This spreading is experimentally relevant in ultracold-atom experiments realizing bosonic Mott insulators, where it was shown to affect the effective on-site interaction [28-31]. We now describe how this delocalization through interactions could be finely resolved using excitationrate measurements.

First, we note that the two-body Schrödinger equation can be decomposed in terms of the center of mass and relative

\footnotetext{
${ }^{2}$ In the case of indistinguishable particles, the protocol would give access to $\operatorname{Var}(\hat{x})$ through $\Gamma_{\hat{x}_{1}+\hat{x}_{2}}^{\text {int }}$.
} 

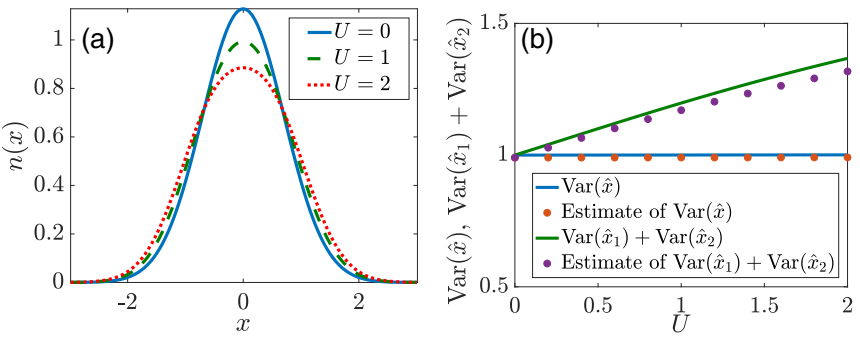

FIG. 3. Two interacting particles in a harmonic trap. (a) Density distribution $n(x)$ in the ground state, for increasing values of the interaction strength $U$ (in units of $\hbar \Omega \sqrt{\hbar / m \Omega}$ ); the position $x$ is expressed in units of $\sqrt{\hbar / m \Omega}$. (b) Spatial variances as extracted from integrated excitation rates (dots) and compared to their exact values (solid lines). The center-of-mass variance $\operatorname{Var}(\hat{x})$ is independent of the interaction strength $U$ (horizontal line). In contrast, the quantity $\operatorname{Var}\left(\hat{x}_{1}\right)+\operatorname{Var}\left(\hat{x}_{2}\right)$ captures well the spreading of the wave function upon increasing the repulsive interaction $U$ (tilted line). Simulations were performed with a modulation strength $E=0.01 \sqrt{m \hbar \Omega^{3}}$ and an observation time $t=5 / \Omega$; the excitation rates were integrated over $\omega$ up to the value $10 \Omega$, using discrete steps of $0.05 \Omega$.

motions. The center of mass is known to be independent of the interparticle interactions [52], and hence the related variance $\operatorname{Var}(\hat{x})=\operatorname{Var}\left(\hat{x}_{1}+\hat{x}_{2}\right)$ does not depend on $U$. In contrast, the density spread in Fig. 3(a) is accurately captured by the relevant quantity $\operatorname{Var}\left(\hat{x}_{1}\right)+\operatorname{Var}\left(\hat{x}_{2}\right)=(1 / 2)\left[\operatorname{Var}\left(\hat{x}_{1}-\hat{x}_{2}\right)+\right.$ $\operatorname{Var}(\hat{x})]$, which is associated with the relative motion and depends on $U$. While $\operatorname{Var}(\hat{x})$ is directly accessible through the driving protocol described above [Eqs. (1)-(4)], the detection of $\operatorname{Var}\left(\hat{x}_{1}-\hat{x}_{2}\right)$ requires a particle-dependent modulation of the form $\hat{V}(t)=2 E\left(\hat{x}_{1}-\hat{x}_{2}\right) \cos (\omega t)$. Such a drive can be realized by considering two atomic internal states with opposite magnetic moments subjected to an oscillating magnetic field $[53,54]$. We describe below how this modification of the driving scheme allows for an accurate evaluation of the two-particle wave-function spreading.

We have numerically calculated the integrated excitation rates $\Gamma_{\hat{x}_{1}-\hat{x}_{2}}^{\mathrm{int}}\left(\Gamma_{\hat{x}_{1}+\hat{x}_{2}}^{\mathrm{int}}\right)$ upon subjecting the two-particle system to the particle-dependent (independent) modulations. From these excitation rates, we can estimate both $\operatorname{Var}(\hat{x})$ and $\operatorname{Var}\left(\hat{x}_{1}\right)+\operatorname{Var}\left(\hat{x}_{2}\right)$. The numerical results presented in Fig. 3(b) show that the variance estimated from excitationrate measurements (dots) perfectly reproduces the exact result (solid line). These simulations confirm that the delocalizationby-interaction effect can be quantitatively measured in ultracold atoms through spectroscopic responses.

Localized spin excitations. The localization of spin excitations, in (disordered) Heisenberg spin chains [55-57], can be detected using this same spectroscopic approach. The modulation $\hat{V}(t)=2 E \cos (\omega t) \sum_{j} j \hat{S}_{j}^{+} \hat{S}_{j}^{-}$should be proportional to the center of mass of the magnonic excitation [58], where $\hat{S}_{j}^{ \pm}$are spin operators on site $j$.

Many-body quantum geometric tensor. Dissipative responses are closely related to the concept of quantum geometry [23-27,35]. At a fundamental level, the geometry of a quantum state $|\psi(\boldsymbol{\theta})\rangle$, which depends on a set of parameters $\boldsymbol{\theta}=\left(\theta_{1}, \theta_{2}, \ldots\right)$, is described by the quantum geometric tensor [35]

$$
\chi_{i j}(\boldsymbol{\theta}) \equiv\left\langle\partial_{\theta_{i}} \psi \mid \partial_{\theta_{j}} \psi\right\rangle-\left\langle\partial_{\theta_{i}} \psi \mid \psi\right\rangle\left\langle\psi \mid \partial_{\theta_{j}} \psi\right\rangle .
$$

Its imaginary part is related to the Berry curvature, $\operatorname{Im} \chi_{i j}=$ $\Omega_{i j} / 2$, which is associated with the physics of the geometric (Berry) phase and topological matter [7,8,47,48,59,60], whereas its real part is known as the quantum metric (or Fubini-Study metric) tensor [34,35,61], $\operatorname{Re} \chi_{i j}=g_{i j}$. The full quantum geometric tensor was recently extracted from Rabi-oscillation measurements in diamond nitrogenvacancy centers [62], from polarization tomography in polaritons [63], and through similar methods in superconducting qubits [64].

The ground state of a many-body Hamiltonian can exhibit nontrivial geometric and topological properties. This can be revealed by introducing twisted boundary conditions [23,3739] and by calculating the quantum geometric tensor (7) in the parameter space spanned by the corresponding twist angles $\boldsymbol{\theta}=\left(\theta_{x}, \theta_{y}, \ldots\right)$. As shown in Ref. [23], the real part of the so-defined quantum geometric tensor, i.e., the manybody quantum metric, describes the variance of the position operator [51]

$$
g_{x x}^{\mathrm{MB}}=\operatorname{Var}(\hat{x}) / L_{x}^{2},
$$

where $L_{x}$ denotes the system's length along $x$.

Combining Eq. (8) with Eq. (4) indicates that the manybody quantum metric $g_{x x}^{\mathrm{MB}}$ is directly accessible through excitation-rate measurements $\left(\Gamma_{\hat{x}}^{\mathrm{int}}\right)$. Similarly, the integrated rate $\Gamma_{\hat{y}}^{\text {int }}$ upon linear shaking along the $y$ direction is proportional to $g_{y y}^{\mathrm{MB}}$; if the modulation is aligned along the diagonal $x+y$ direction, the resulting integrated rate is proportional to $L_{x}^{2} g_{x x}^{\mathrm{MB}}+2 L_{x} L_{y} g_{x y}^{\mathrm{MB}}+L_{y}^{2} g_{y y}^{\mathrm{MB}}$. Hence, all the components of the many-body quantum metric can be extracted from excitation-rate measurements upon linear shaking.

On the other hand, the many-body Berry curvature is related to the integrated rate upon circular shaking [45]. Indeed, considering the periodic modulation $\hat{V}_{ \pm}(t)=2 E[\hat{x} \cos (\omega t) \pm$ $\hat{y} \sin (\omega t)]$, the integrated rates read

$$
\begin{aligned}
\Gamma_{ \pm}^{\mathrm{int}} & =\frac{2 \pi E^{2}}{\hbar^{2}} \sum_{\beta \neq \alpha}|\langle\beta|\hat{x} \mp i \hat{y}| \alpha\rangle|^{2} \\
& =\frac{2 \pi E^{2}}{\hbar^{2}}\left(L_{x}^{2} g_{x x}^{\mathrm{MB}}+L_{y}^{2} g_{y y}^{\mathrm{MB}} \pm L_{x} L_{y} \Omega_{x y}^{\mathrm{MB}}\right) .
\end{aligned}
$$

Therefore, the many-body Berry curvature is given by the differential integrated rate per unit area

$$
\left(\Gamma_{+}^{\mathrm{int}}-\Gamma_{-}^{\mathrm{int}}\right) / 2 L_{x} L_{y}=\frac{2 \pi E^{2}}{\hbar^{2}} \Omega_{x y}^{\mathrm{MB}} .
$$

This relation between circular dichroism and the many-body Berry curvature (or nonintegrated Chern number [39]) can also be derived from Kramers-Kronig relations [45].

Summarizing, all the components of the many-body quantum geometric tensor are related to an observable response of the system upon linear or circular shaking. This result generalizes previous connections between the quantum geometry of single-particle states and spectroscopic responses [24-27] to a many-body framework. In the special case of a band insulator, the many-body quantum metric is proportional to the average 
of the single-particle quantum metric over the entire Brillouin zone [51].

Conclusion. This work proposed spectroscopic responses as a method to study localization in quantum many-body systems, offering a practical alternative to in situ imaging. It is intriguing to observe that such excitation-rate measurements can extract information on both geometry and localization, two important concepts in condensed matter, through the extraction of the many-body quantum geometric tensor. An exciting perspective concerns the application of the present approach to explore the localization properties of many-body quantum states of interest, such as excitations of fractional quantum Hall liquids $[65,66]$, many-body localized systems $[10,11]$, and fractons [67].

Acknowledgments. We are grateful for insightful discussions with Cécile Repellin, Monika Aidelsburger, and Yoshiro Takahashi. T.O. was supported by JSPS KAKENHI Grant No. JP18H05857, RIKEN Incentive Research Project, and the Interdisciplinary Theoretical and Mathematical Sciences Program at RIKEN. N.G. was supported by the FRS-FNRS (Belgium) and the ERC Starting Grant TopoCold.
[1] P. W. Anderson, Absence of diffusion in certain random lattices, Phys. Rev. 109, 1492 (1958).

[2] P. A. Lee and T. V. Ramakrishnan, Disordered electronic systems, Rev. Mod. Phys. 57, 287 (1985).

[3] B. Kramer and A. MacKinnon, Localization: theory and experiment, Rep. Prog. Phys. 56, 1469 (1993).

[4] C. W. J. Beenakker, Random-matrix theory of quantum transport, Rev. Mod. Phys. 69, 731 (1997).

[5] E. K. Kudinov, Difference between insulating and conducting states, Fis. Tverd. Tela 33, 2306 (1991) [Sov. Phys. Solid State 33, 1299 (1991)].

[6] R. Resta and S. Sorella, Electron Localization in the Insulating State, Phys. Rev. Lett. 82, 370 (1999).

[7] M. Z. Hasan and C. L. Kane, Colloquium: Topological insulators, Rev. Mod. Phys. 82, 3045 (2010).

[8] X.-L. Qi and S.-C. Zhang, Topological insulators and superconductors, Rev. Mod. Phys. 83, 1057 (2011).

[9] D. M. Basko, I. L. Aleiner, and B. L. Altshuler, Metal-insulator transition in a weakly interacting many-electron system with localized single-particle states, Ann. Phys. (NY) 321, 1126 (2006).

[10] R. Nandkishore and D. A. Huse, Many-body localization and thermalization in quantum statistical mechanics, Annu. Rev. Condens. Matter Phys. 6, 15 (2015).

[11] D. A. Abanin, E. Altman, I. Bloch, and M. Serbyn, Colloquium: Many-body localization, thermalization, and entanglement, Rev. Mod. Phys. 91, 021001 (2019).

[12] J. Billy, V. Josse, Z. Zuo, A. Bernard, B. Hambrecht, P. Lugan, D. Clément, L. Sanchez-Palencia, P. Bouyer, and A. Aspect, Direct observation of Anderson localization of matter waves in a controlled disorder, Nature (London) 453, 891 (2008).

[13] G. Roati, C. D’Errico, L. Fallani, M. Fattori, C. Fort, M. Zaccanti, G. Modugno, M. Modugno, and M. Inguscio, Anderson localization of a non-interacting Bose-Einstein condensate, Nature (London) 453, 895 (2008).

[14] S. S. Kondov, W. R. McGehee, J. J. Zirbel, and B. DeMarco, Three-dimensional Anderson localization of ultracold matter, Science 334, 66 (2011)

[15] M. Schreiber, S. S. Hodgman, P. Bordia, H. P. Lüschen, M. H. Fischer, R. Vosk, E. Altman, U. Schneider, and I. Bloch, Observation of many-body localization of interacting fermions in a quasirandom optical lattice, Science 349, 842 (2015).

[16] J. Choi, S. Hild, J. Zeiher, P. Schauß, A. Rubio-Abadal, T. Yefsah, V. Khemani, D. A. Huse, I. Bloch, and C. Gross,
Exploring the many-body localization transition in two dimensions, Science 352, 1547 (2016).

[17] J. Smith, A. Lee, P. Richerme, B. Neyenhuis, P. W. Hess, P. Hauke, M. Heyl, D. A. Huse, and C. Monroe, Many-body localization in a quantum simulator with programmable random disorder, Nat. Phys. 12, 907 (2016).

[18] P. Roushan, C. Neill, J. Tangpanitanon, V. M. Bastidas, A. Megrant, R. Barends, Y. Chen, Z. Chen, B. Chiaro, A. Dunsworth et al., Spectroscopic signatures of localization with interacting photons in superconducting qubits, Science $\mathbf{3 5 8}$ 1175 (2017).

[19] A. Lukin, M. Rispoli, R. Schittko, M. E. Tai, A. M. Kaufman, S. Choi, V. Khemani, J. Léonard, and M. Greiner, Probing entanglement in a many-body-localized system, Science 364, 256 (2019)

[20] H. B. Callen and T. A. Welton, Irreversibility and generalized noise, Phys. Rev. 83, 34 (1951).

[21] R. Kubo, Statistical-mechanical theory of irreversible processes. I. General theory and simple applications to magnetic and conduction problems, J. Phys. Soc. Jpn. 12, 570 (1957).

[22] L. D. Landau and E. M. Lifshitz, Statistical Physics, 3rd ed. (Butterworth-Heinemann, Oxford, 1980), Pt. 1.

[23] I. Souza, T. Wilkens, and R. M. Martin, Polarization and localization in insulators: Generating function approach, Phys. Rev. B 62, 1666 (2000).

[24] L. Asteria, D. T. Tran, T. Ozawa, M. Tarnowski, B. S. Rem, N. Fläschner, K. Sengstock, N. Goldman, and C. Weitenberg, Measuring quantized circular dichroism in ultracold topological matter, Nat. Phys. 15, 449 (2019).

[25] D. T. Tran, A. Dauphin, A. G. Grushin, P. Zoller, and N. Goldman, Probing topology by "heating": Quantized circular dichroism in ultracold atoms, Sci. Adv. 3, e1701207 (2017).

[26] D. T. Tran, N. R. Cooper, and N. Goldman, Quantized Rabi oscillations and circular dichroism in quantum Hall systems, Phys. Rev. A 97, 061602(R) (2018).

[27] T. Ozawa and N. Goldman, Extracting the quantum metric tensor through periodic driving, Phys. Rev. B 97, 201117(R) (2018).

[28] G. K. Campbell, J. Mun, M. Boyd, P. Medley, A. E. Leanhardt, L. G. Marcassa, D. E. Pritchard, and W. Ketterle, Imaging the Mott insulator shells by using atomic clock shifts, Science 313, 649 (2006)

[29] S. Will, T. Best, U. Schneider, L. Hackermüller, D.-S Lühmann, and I. Bloch, Time-resolved observation of coherent 
multi-body interactions in quantum phase revivals, Nature (London) 465, 197 (2010).

[30] S. Will, T. Best, S. Braun, U. Schneider, and I. Bloch, Coherent Interaction of a Single Fermion with a Small Bosonic Field, Phys. Rev. Lett. 106, 115305 (2011).

[31] G. Zürn, F. Serwane, T. Lompe, A. N. Wenz, M. G. Ries, J. E. Bohn, and S. Jochim, Fermionization of Two Distinguishable Fermions, Phys. Rev. Lett. 108, 075303 (2012).

[32] S. Subhankar, Y. Wang, T.-C. Tsui, S. L. Rolston, and J. V. Porto, Nanoscale Atomic Density Microscopy, Phys. Rev. X 9, 021002 (2019).

[33] M. McDonald, J. Trisnadi, K.-X. Yao, and C. Chin, Superresolution Microscopy of Cold Atoms in an Optical Lattice, Phys. Rev. X 9, 021001 (2019).

[34] J. P. Provost and G. Vallee, Riemannian structure on manifolds of quantum states, Commun. Math. Phys. 76, 289 (1980).

[35] M. Kolodrubetz, D. Sels, P. Mehta, and A. Polkovnikov, Geometry and non-adiabatic response in quantum and classical systems, Phys. Rep. 697, 1 (2017).

[36] P. Hauke, M. Heyl, L. Tagliacozzo, and P. Zoller, Measuring multipartite entanglement through dynamic susceptibilities, Nat. Phys. 12, 778 (2016).

[37] Q. Niu, D. J. Thouless, and Y.-S. Wu, Quantized Hall conductance as a topological invariant, Phys. Rev. B 31, 3372 (1985).

[38] H. Watanabe, Insensitivity of bulk properties to the twisted boundary condition, Phys. Rev. B 98, 155137 (2018).

[39] K. Kudo, H. Watanabe, T. Kariyado, and Y. Hatsugai, ManyBody Chern Number without Integration, Phys. Rev. Lett. 122, 146601 (2019).

[40] J. J. Sakurai, Modern Quantum Mechanics, 2nd ed. (Cambridge University Press, Cambridge, 2017).

[41] M. Aidelsburger, M. Lohse, C. Schweizer, M. Atala, J. T. Barreiro, S. Nascimbène, N. R. Cooper, I. Bloch, and N. Goldman, Measuring the Chern number of Hofstadter bands with ultracold bosonic atoms, Nat. Phys. 11, 162 (2015).

[42] G. Jotzu, M. Messer, F. Görg, D. Greif, R. Desbuquois, and T. Esslinger, Creating State-Dependent Lattices for Ultracold Fermions by Magnetic Gradient Modulation, Phys. Rev. Lett. 115, 073002 (2015).

[43] M. Reitter, J. Näger, K. Wintersperger, C. Sträter, I. Bloch, A. Eckardt, and U. Schneider, Interaction Dependent Heating and Atom Loss in a Periodically Driven Optical Lattice, Phys. Rev. Lett. 119, 200402 (2017).

[44] T. Boulier, J. Maslek, M. Bukov, C. Bracamontes, E. Magnan, S. Lellouch, E. Demler, N. Goldman, and J. V. Porto, Parametric Heating in a 2D Periodically Driven Bosonic System: Beyond the Weakly Interacting Regime, Phys. Rev. X 9, 011047 (2019).

[45] C. Repellin and N. Goldman, Detecting Fractional Chern Insulators through Circular Dichroism, Phys. Rev. Lett. 122, 166801 (2019).

[46] N. Goldman, J. Beugnon, and F. Gerbier, Detecting Chiral Edge States in the Hofstadter Optical Lattice, Phys. Rev. Lett. 108, 255303 (2012).

[47] D. Xiao, M.-C. Chang, and Q. Niu, Berry phase effects on electronic properties, Rev. Mod. Phys. 82, 1959 (2010).

[48] N. R. Cooper, J. Dalibard, and I. B. Spielman, Topological bands for ultracold atoms, Rev. Mod. Phys. 91, 015005 (2019).

[49] Y. Hatsugai, Quantized Berry phases as a local order parameter of a quantum liquid, J. Phys. Soc. Jpn. 75, 123601 (2006).
[50] M. Leder, C. Grossert, L. Sitta, M. Genske, A. Rosch, and M. Weitz, Real-space imaging of a topologically protected edge state with ultracold atoms in an amplitude-chirped optical lattice, Nat. Commun. 7, 13112 (2016).

[51] See Supplemental Material at http://link.aps.org/supplemental/ 10.1103/PhysRevResearch.1.032019 for the case of a system of a single particle moving in a one-dimensional harmonic trap, derivation of Eq. (8), and the relation between the many-body quantum metric and the quantum metric of Bloch bands.

[52] T. Busch, B.-G. Englert, K. Rzażewski, and M. Wilkens, Two cold atoms in a harmonic trap, Found. Phys. 28, 549 (1998).

[53] M. Aidelsburger, M. Atala, M. Lohse, J. T. Barreiro, B. Paredes, and I. Bloch, Realization of the Hofstadter Hamiltonian with Ultracold Atoms in Optical Lattices, Phys. Rev. Lett. 111, 185301 (2013).

[54] C. J. Kennedy, G. A. Siviloglou, H. Miyake, W. C. Burton, and W. Ketterle, Spin-Orbit Coupling and Quantum Spin Hall Effect for Neutral Atoms without Spin Flips, Phys. Rev. Lett. 111, 225301 (2013).

[55] S. K. Lyo, Magnon Localization in Antiferromagnets, Phys. Rev. Lett. 28, 1192 (1972).

[56] J.-i. Igarashi, Anderson localization of spin waves in random Heisenberg antiferromagnets, Phys. Rev. B 35, 5151 (1987).

[57] B. Y. Pan, S. Y. Zhou, X. C. Hong, X. Qiu, and S. Y. Li, Anderson localization of spinons in a spin-1/2 antiferromagnetic Heisenberg chain, arXiv:1208.3803.

[58] T. Matsubara and H. Matsuda, A lattice model of liquid helium, I, Prog. Theor. Phys. 16, 569 (1956)

[59] C. A. Mead, The geometric phase in molecular systems, Rev. Mod. Phys. 64, 51 (1992).

[60] T. Ozawa, H. M. Price, A. Amo, N. Goldman, M. Hafezi, L. Lu, M. C. Rechtsman, D. Schuster, J. Simon, O. Zilberberg, and I. Carusotto, Topological photonics, Rev. Mod. Phys. 91, 015006 (2019).

[61] J. Anandan and Y. Aharonov, Geometry of Quantum Evolution, Phys. Rev. Lett. 65, 1697 (1990).

[62] M. Yu, P. Yang, M. Gong, Q. Cao, Q. Lu, H. Liu, M. B. Plenio, F. Jelezko, T. Ozawa, N. Goldman, S. Zhang, and J. Cai, Experimental measurement of the complete quantum geometry of a solid-state spin system, arXiv:1811.12840.

[63] A. Gianfrate, O. Bleu, L. Dominici, V. Ardizzone, M. De Giorgi, D. Ballarini, K. West, L. N. Pfeiffer, D. D. Solnyshkov, D. Sanvitto, and G. Malpuech, Direct measurement of the quantum geometric tensor in a two-dimensional continuous medium, arXiv:1901.03219.

[64] X. Tan, D.-W. Zhang, Z. Yang, J. Chu, Y.-Q. Zhu, D. Li, X. Yang, S. Song, Z. Han, Z. Li, Y. Dong, H.-F. Yu, H. Yan, S.-L. Zhu, and Y. Yu, Experimental Measurement of the Quantum Metric Tensor and Related Topological Phase Transition with a Superconducting Qubit, Phys. Rev. Lett. 122, 210401 (2019)

[65] D. Yoshioka, The Quantum Hall Effect, 1st ed. (Springer, Berlin, 2002).

[66] J. Martin, S. Ilani, B. Verdene, J. Smet, V. Umansky, D. Mahalu, D. Schuh, G. Abstreiter, and A. Yacoby, Localization of fractionally charged quasi-particles, Science 305, 980 (2004).

[67] R. M. Nandkishore and M. Hermele, Fractons, Annu. Rev. Condens. Matter Phys. 10, 295 (2019). 